\title{
Synteny of human chromosomes 14 and 15 in the platyrrhines (Primates, Platyrrhinı)
}

\author{
Cristiani Gifalli-Iughetti and Célia P. Koiffmann \\ Centro de Estudos do Genoma Humano, Departamento de Genética e Biologia Evolutiva, Instituto de \\ Biociências, Universidade de São Paulo, São Paulo, SP, Brazil.
}

\begin{abstract}
In order to study the intra- and interspecific variability of the 14/15 association in Platyrrhini, we analyzed 15 species from 13 genera, including species that had not been described yet. The DNA libraries of human chromosomes 14 and 15 were hybridized to metaphases of Alouatta guariba clamitans, A. caraya, A. sara, Ateles paniscus chamek, Lagothrix lagothricha, Brachyteles arachnoides, Saguinus midas midas, Leontopithecus chrysomelas, Callimico goeldii, Callithrix sp., Cebus apella, Aotus nigriceps, Cacajao melanocephalus, Chiropotes satanas and Callicebus caligatus. The 14/15 hybridization pattern was present in 13 species, but not in Alouatta sara that showed a 14/15/14 pattern and Aotus nigriceps that showed a 15/14/15/14 pattern. In the majority of the species, the HSA 14 homologue retained synteny for the entire chromosome, whereas the HSA 15 homologue displayed fragmented segments. Within primates, the New World monkeys represent the taxon with the highest variability in chromosome number $(2 n=16$ to 62$)$. The presence of the HSA 14/15 association in all species and subspecies studied herein confirms that this association is the ancestral condition for platyrrhines and that this association has been retained in most platyrrhines, despite the occurrence of extensive inter- and intrachromosomal rearrangements in this infraorder of Primates.
\end{abstract}

Key words: HSA 14/15 synteny, homologies, chromosomal evolution, Platyrrhini, New World monkeys.

Received: October 16, 2008; Accepted: March 11, 2009.

The neighboring association of human chromosome 14 and 15 segments is a well-known ancestral mammalian synteny observed in primates and non-primates and is an important evolutionary landmark (Ruiz-Herrera et al., 2005a). In the majority of cases, the association involves conserved syntenies of the complete human chromosomes (Frönicke, 2005). Therefore, the two human chromosomes clearly represent one ancestral eutherian chromosome (Chowdhary et al., 1998). This association was found in all Zoo-FISH studies except in Canis familiaris (Canidae, Carnivora) (Breen et al., 1999), Ailuropoda melanoleuca (Ursidae, Carnivora) (Nash et al., 1998), Alouatta sara and A. arctoidea (Atelidae, Platyrrhini, Primates) (Consigliere et al., 1996), and in lesser apes and great apes (Jauch et al., 1992; Haig, 1999; Wienberg, 2005; Ferguson-Smith and Trifonov, 2007).

In all Platyrrhini (New World monkeys, Primates) species that have been investigated with whole human chromosome 14 and 15 paints (WCP), conserved segments of the entire HSA (Homo sapiens) 14 and fissioning of

Send correspondence to Cristiani Gifalli lughetti. Centro de Estudos do Genoma Humano, Departamento de Genética e Biologia Evolutiva, Instituto de Biociências, Universidade de São Paulo, Rua do Matão 277, Butantã, 05508-090 São Paulo, SP, Brazil. E-mail: criscg@ib.usp.br.
HSA 15 were observed, although the 14/15 association was still conserved (Seuánez et al., 1994; Consigliere et al., 1996; Richard et al., 1996; Morescalchi et al., 1997; Consigliere et al., 1998; García et al., 1999, 2000; Stanyon et al., 2000; Mudry et al., 2001; Müller et al., 2001; Neusser et al., 2001; Stanyon et al., 2001; De Oliveira et al., 2002; García et al., 2002; Barros et al., 2003; Stanyon et al., 2003; Gerbault-Serreau et al., 2004). Moreover, the $14 / 15$ association was also present in Saimiri (Stanyon et al., 2000), Cebus (García et al., 2002) and Aotus (Stanyon et al., 2004; Ruiz-Herrera et al., 2005b), although it appears to have undergone intrachromosomal reorganizations.

In order to study the intra- and interspecific variability of the 14/15 syntenic association in Platyrrhini, we analyzed 15 species from 13 genera and described for the first time the HSA 14/15 association in the species Ateles paniscus chamek (Atelidae), Saguinus midas midas and Aotus nigriceps (Cebidae), Cacajao melanocephalus, Chiropotes satanas, and Callicebus caligatus (Pithecidae). We also confirmed the association of these chromosomes in nine previously described species of platyrrhines: Alouatta sara, A. guariba clamitans, A. caraya, Lagothrix lagothricha, and Brachyteles arachnoides (Atelidae), Leontopithecus chrysomelas, Callimico goeldii, Callithrix sp., and Cebus apella (Cebidae). 
Using standard protocols for blood cell and lymphocyte cultures, metaphases were obtained from females of the species Alouatta guariba clamitans, A. caraya, Lagothrix lagothricha, Saguinus midas midas, Leontopithecus chrysomelas, Callimico goeldii, Aotus nigriceps, Chiropotes satanas, and Callicebus caligatus and from males of the species Alouatta sara, Ateles paniscus chamek, Brachyteles arachnoides, Callithrix sp., Cebus apella, and Cacajao melanocephalus. Chromosomes were prepared and stored in fixative at $-20{ }^{\circ} \mathrm{C}$. Lagothrix lagothricha, Ateles paniscus chamek, and Cebus apella are kept at Fundação Parque Zoológico de São Paulo (São Paulo, SP, Brazil); Saguinus midas midas, Leontopithecus chrysomelas, Callimico goeldii, Aotus nigriceps, and Brachyteles arachnoides at Zoológico Municipal Quinzinho de Barros (Sorocaba, SP, Brazil); Callicebus caligatus at Zôo Parque de Itatiba (Itatiba, SP, Brazil); Cacajao melanocephalus at Centro de Primatologia do Rio de Janeiro (Rio de Janeiro, RJ, Brazil); Callithrix sp. at Barragem Paraitinga (Salesópolis, SP, Brazil); Chiropotes satanas and Alouatta sara at Fundação Zôo-Botânica de Belo Horizonte (Belo Horizonte, MG, Brazil); A. guariba clamitans at Departamento de Parques e Áreas Verdes do Estado de São Paulo, Divisão Técnica de Medicina Veterinária e Manejo da Fauna Silvestre (São Paulo, SP, Brazil); and A. caraya at Presidente Epitácio (Porto Primavera, SP, Brazil).

Fluorescent in situ hybridization (FISH) with WCP 14 and 15 (Oncor, USA) was carried out according to the manufacturer's protocol. Chromosome preparations were hybridized for two to seven days at $37^{\circ} \mathrm{C}$. Chromosomes were counterstained in blue with DAPI (4,6 diamidino2-phenylindole) and Vectashield (Switzerland). Metaphase images were obtained with a CCD camera coupled to a Zeiss Axiophot 2 motorized microscope and analyzed with the MetaSystem Isis software (USA).

In the karyotype of one female specimen of Alouatta guariba clamitans $(2 \mathrm{n}=50)$ from the State of São Paulo, Brazil, the HSA 14 homologue (red) was located in the distal region of the long arm of a large submetacentric pair of chromosomes, juxtaposed to a WCP 15 signal $\left(15 \mathrm{a}_{1}\right.$, green). Other signals produced by HSA 15 painting were found in the distal region of the long arm of the typical large submetacentric pair of $A$. guariba clamitans $\left(15 \mathrm{a}_{2}\right)$ and on the short arm of a medium-sized submetacentric pair (15b). De Oliveira et al. (2002) described two male specimens from the State of Paraná with $2 \mathrm{n}=45$, and in both WCP 14 hybridized to the same large submetacentric pair, whereas the human WCP 15 labeled two autosomal pairs (15a $\mathrm{a}_{1}$ and $15 \mathrm{a}_{2}$ ) and chromosomes $\mathrm{X}_{2}$ and $\mathrm{Y}_{1}(15 \mathrm{~b})$ (for males), as in our female.

In Alouatta caraya $(2 \mathrm{n}=52)$, the HSA 14 homologue (red) was found in the distal region of the long arm of a medium-sized acrocentric pair, juxtaposed to a proximal region painted by WCP $15\left(15 \mathrm{a}_{1}\right.$, green). The other homo- logies with HSA 15 were found on the short arm of a medium-sized submetacentric pair $\left(15 \mathrm{~b}, \mathrm{X}_{2}\right)$ and on the long arm of a medium-sized acrocentric pair $\left(15 \mathrm{a}_{2}\right)$. A male and a female of Alouatta caraya $(2 \mathrm{n}=52)$ analyzed by De Oliveira et al. (2002) displayed the same hybridized pairs as our specimen, except for a pair of acrocentric chromosomes painted in the proximal region, whereas in our specimen the labeled region $\left(15 \mathrm{a}_{2}\right)$ was distal.

In Alouatta sara $(2 \mathrm{n}=50)$ (Figure 1a), WCP 14 (red) was found to be present along almost the entire length of a medium-sized submetacentric pair, except for the proximal region of the long arm that was painted by WCP $15\left(15 \mathrm{a}_{1}\right.$, green), showing a 14/15/14 hybridization pattern. Two other pairs were also painted by WCP 15 , one a heteromorphic pair (15b, the submetacentric chromosome with the signal across the short arm and the acrocentric chromosome with the signal in the distal region of the long arm), and the other a large acrocentric pair $\left(15 \mathrm{a}_{2}\right.$, in the distal region of the long arm). The heteromorphic pair hybridized by WCP 15 corresponds to the sex chromosomes $\mathrm{X}_{2}$ (originally a submetacentric autosome) and $\mathrm{Y}_{1}$ (a translocation product of the original $\mathrm{Y}$ chromosome), resulting from a $\mathrm{Y}$-autosome translocation that is rare in primates, except in Platyrrhini (Stanyon et al., 1995; Consigliere et al., 1996). The HSA $14 / 15$ association in $A$. $\operatorname{sara}(2 \mathrm{n}=50)$ was not ob-

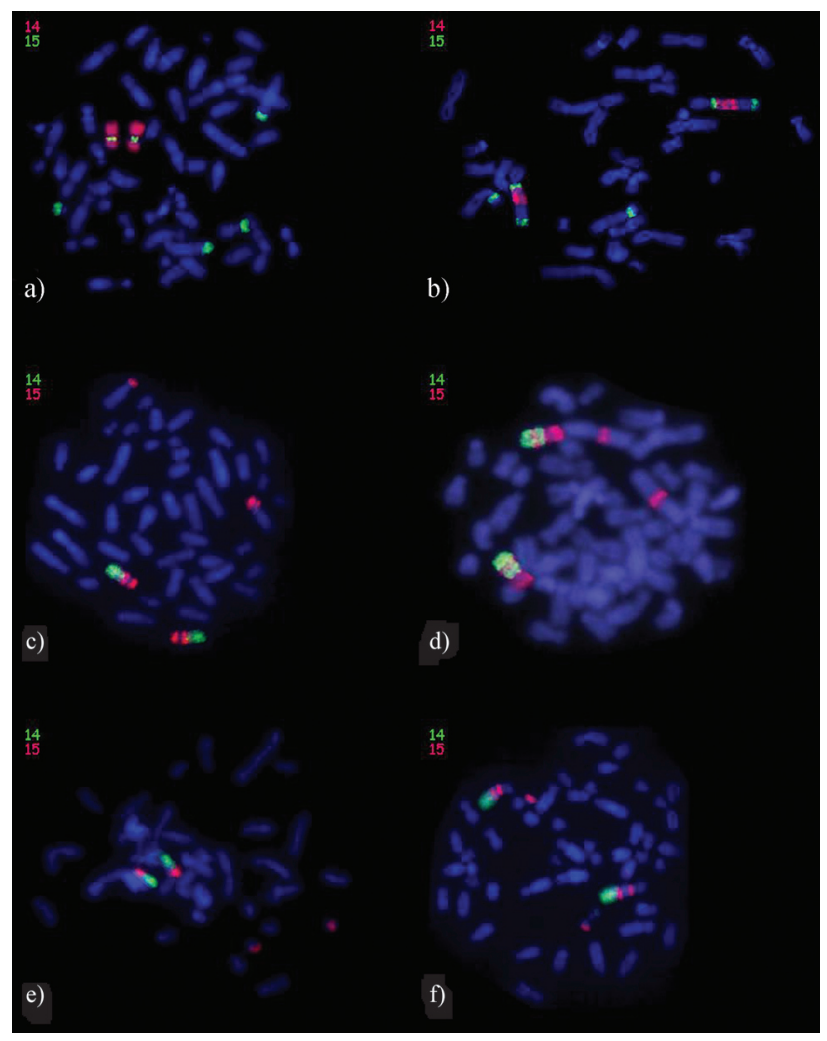

Figure 1 - In situ hybridization of human chromosome 14 and 15 whole chromosome probes (WCP) on metaphases of: (a) Alouatta sara, (b) Ateles paniscus chamek, (c) Saguinus midas midas, (d) Aotus nigriceps, (e) Cacajao melanocephalus, and (f) Chiropotes satanas. In (a) and (b): WCP 14 - red, WCP 15 - green; in (c-f): WCP 14 - green, WCP 15 - red. 
served by Consigliere et al. (1996), probably because the signal that identifies the segment homologous to HSA 15, that is located between the WCP 14 signals, is small and difficult to visualize.

In Ateles paniscus chamek $(2 \mathrm{n}=34)$, the HSA 14 homologue (red) was located on the long arm of a large submetacentric pair, juxtaposed to a proximal region painted by WCP 15 ( $15 \mathrm{a}_{1}$, green). Another WCP 15 signal was detected in the distal region of the long arm of the same chromosome $\left(15 \mathrm{a}_{2}\right)$. A third signal produced by HSA 15 painting was detected in the proximal region of the short arm of a medium-sized submetacentric pair (15b) (Figure $1 b)$. In previous studies, $A$. p. paniscus $(2 \mathrm{n}=32), A$. belzebuth marginatus $(2 \mathrm{n}=34)$ (De Oliveira et al., 2005), A. b. hybridus $(2 \mathrm{n}=34)$ (García et al., 2002), and $A$. geoffroyi $(2 \mathrm{n}=34)$ (Morescalchi et al., 1997) had shown the same hybridized chromosome pairs as our A. paniscus chamek specimen. The drastic reduction in the diploid numbers of Ateles $(2 \mathrm{n}=32,34)$, compared to other Atelidae genera, has been explained as resulting from several chromosomal rearrangements, including translocations and in tandem fusions. These rearrangements also include the HSA 15 homologue, since two WCP 15 signals were detected on a large submetacentric pair $\left(15 \mathrm{a}_{1}\right.$ and $\left.15 \mathrm{a}_{2}\right)$, whereas these signals were split onto two different chromosomes in other Atelidae species.

In Lagothrix lagothricha $(2 \mathrm{n}=62)$, and Brachyteles arachnoides $(2 \mathrm{n}=62)$, WCP 14 (red) painted the long arm of a medium-sized submetacentric pair, juxtaposed to a WCP 15 signal $\left(15 \mathrm{a}_{1}\right.$, green) on the short arm. Almost the entire long arm of a small acrocentric pair (15b) and the distal region of a medium-sized acrocentric pair also hybridized to WCP $15\left(15 \mathrm{a}_{2}\right)$. Stanyon et al. (2001) studied a female of Lagothrix lagothricha $(2 \mathrm{n}=62)$ and De Oliveira et al. (2005) studied a male of Brachyteles arachnoides $(2 n=62)$ and differences in the morphology of the labeled pairs were observed: in L. lagothricha, the chromosome pair with the 14/15 association (pair 21) was acrocentric, whereas in B. arachnoides it was submetacentric (pair 9). Also in Lagothrix, an acrocentric pair was labeled by WCP 15 in the distal region, whereas in Brachyteles it was labeled in the proximal region $\left(15 \mathrm{a}_{2}\right)$, probably indicating an inversion process.

In the Atelidae family species studied herein, the HSA 15 homologue was divided into six blocks located on three chromosome pairs in Alouatta guariba clamitans, $A$. caraya, A. sara, Lagothrix lagothricha, and Brachyteles arachnoides, and on two chromosome pairs in Ateles paniscus chamek.

In the Callitrichinae (Cebidae) subfamily species studied herein, Saguinus midas midas $(2 \mathrm{n}=46)$ (Figure 1c), Leontopithecus chrysomelas $(2 \mathrm{n}=46)$, Callithrix $s p$. $(2 \mathrm{n}=46)$ and Callimico goeldii $(2 \mathrm{n}=48)$, we observed that WCP 14 (green) was located in the distal region of the long arm of a medium-sized submetacentric pair, while the prox- imal region of the long arm as well as the entire short arm (except for the centromeric region) were painted by WCP 15 (15a, red). The short arm of a medium-sized submetacentric pair was also painted by WCP 15 (15b), except for Callimico goeldii that had the last WCP 15 signal in the proximal region of an acrocentric pair (15b). The Callimico goeldii $(2 \mathrm{n}=47)$ specimen reported by Neusser et al. (2001) also presented the WCP 15 signals in the proximal region of an acrocentric pair (15b) and on the submetacentric pair, associated with WCP 14 (14/15a). In Saguinus oedipus $(2 \mathrm{n}=46)$ (Müller et al., 2001), Leontopithecus chrysomelas $(2 \mathrm{n}=46)$ (Gerbault-Serreau et al., 2004), Callithrix jacchus $(2 \mathrm{n}=46)$ (Sherlock et al., 1996; Neusser et al., 2001), and C. argentata $(2 \mathrm{n}=44)$ (Neusser et al., 2001), the same submetacentric pairs hybridized to WCP 14 and 15.

In Cebus apella $(2 \mathrm{n}=54)$ (Cebidae), WCP 14 (green) painted the distal region of the long arm of a medium-sized submetacentric pair. The WCP 15 signals (red) were juxtaposed to WCP 14 in the proximal region of the long arm and on the short arm of the same chromosome pair, except in the centromeric region (15a). In the distal region of a small acrocentric pair, we observed another WCP 15 signal (15b). García et al. (2000) reported for the same species a 14/15/14 hybridization pattern on a submetacentric pair (pair 6) and a WCP 15 signal on an acrocentric pair. The pattern of the submetacentric chromosome pair could be due to a chromosomal inversion.

In Aotus nigriceps $(2 \mathrm{n}=51)$ (Cebidae), WPC 14 (green) painted the long arm of a medium-sized submetacentric pair, except for a small region painted by WCP 15 $\left(15 \mathrm{a}_{2}\right)$ that also hybridized to the short arm of the same chromosome (15a $\mathrm{a}_{1}$ ), showing a 15/14/15/14 hybridization pattern. Another WCP 15 signal was located in a region near the centromere on the long arm of the largest submetacentric pair (15b) (Figure 1d). Stanyon et al. (2004) and Ruiz-Herrera et al. $(2005 \mathrm{~b})$ studied A. nancymae $(2 \mathrm{n}=54)$ and found that the paint specific for HSA 14 was split into three segments on two chromosome pairs, whereas the paint specific for HSA 15 was located in six segments of different chromosomes per haploid set; the 14/15 association was found on two chromosomes, and a 14/15/14 hybridization pattern was found on a submetacentric pair (pair 14). In Aotus sp., reported by Ruiz-Herrera et al. (2005b) $(2 \mathrm{n}=50)$, WCP 14 labeled one pair, whereas WCP 15 was split into six segments of five chromosome pairs, and a 15/14/15/14 hybridization pattern was seen. According to the studies available to date, Aotus appears to represent a highly diverse group with several specific chromosomal variations, and with respect to the ancestral New World monkey karyotype, this genus presents a highly derived situation.

In Cacajao melanocephalus $(2 \mathrm{n}=45)$, WCP 14 (green) hybridized to the long arm of a medium-sized submetacentric pair, while WCP 15 (red) painted the short 


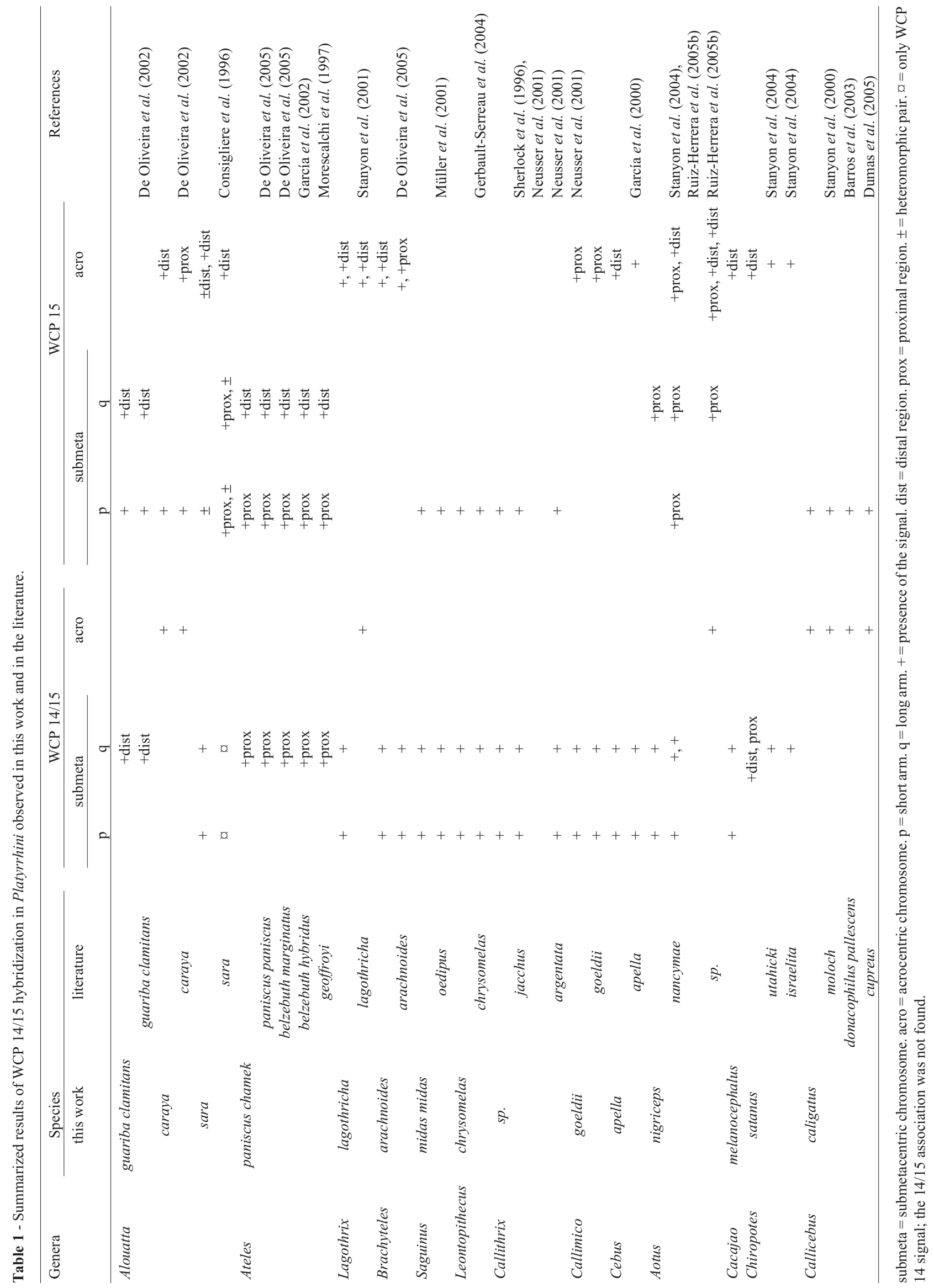


arm of this chromosome (15a) as well as the distal region of the long arm of a small acrocentric pair (15b) (Figure 1e). The presence of the HSA 14/15 association in the genus Cacajao had not been previously reported.

In Callicebus caligatus $(2 \mathrm{n}=48$, this work), C. moloch $(2 \mathrm{n}=50)$ (Stanyon et al., 2000), C. donacophilus pallescens $(2 \mathrm{n}=50)$ (Barros et al., 2003), and C. cupreus $(2 \mathrm{n}=46)$ (Dumas et al., 2005), WCP 14 (green) hybridized to the proximal region of an acrocentric pair, whereas WCP 15 labeled the distal region of the same chromosomes (15a) and also the short arm of a medium-sized submetacentric pair (15b). Even in C. lugens with its 16 chromosomes (Stanyon et al., 2003), WCP 14 produced a signal on one chromosome pair and WCP 15 on two.

In Chiropotes satanas $(2 \mathrm{n}=54)$, WCP 14 (green) painted the distal region of the long arm of the largest submetacentric pair. The WCP 15 signals (red) were juxtaposed to WCP $14\left(15 \mathrm{a}_{1}\right)$ and located in the proximal region of the long arm of the same chromosome $\left(15 \mathrm{a}_{2}\right)$. The distance between these two WCP 15 signals was different for the two homologues, probably because of an inversion process. Another WCP 15 signal was identified in the distal region of a small acrocentric pair (15b) (Figure 1f). In $C$. utahicki $(2 \mathrm{n}=54)$ and $C$. israelita $(2 \mathrm{n}=54)$ (Stanyon et al., 2004), the same painting pattern had been observed, and they apparently showed the same hybridized chromosome pairs as $C$. satanas, except for the large submetacentric pair that displayed a single block homologous to HSA 15.

In the Pithecidae family species studied herein, we observed two chromosomes hybridized by WCP 15, showing four signals in Cacajao melanocephalus and Callicebus caligatus, and six signals in Chiropotes satanas, due to the presence of two separate signals on the same chromosome arm.

Table 1 summarizes the results obtained by hybridizing human WCP 14 and 15 to the chromosomes of the species we analyzed and to those that have been described in literature.

Within primates, the Platyrrhini infraorder represents the group with the greatest variability in chromosome number, ranging from $2 \mathrm{n}=16$ in Callicebus lugens to $2 \mathrm{n}=62$ in Brachyteles and Lagothrix. A 14/15 hybridization pattern was observed in 13 species of 13 genera, the exceptions being Alouatta sara, which showed a $14 / 15 / 14$ pattern, and Aotus nigriceps, which showed a 15/14/15/14 pattern. In all of the species, the HSA 14 homologue retained synteny for the entire chromosome, except for $A$. nancymae, whereas the HSA 15 homologue underwent fragmentations, leading to a shared derived fission of the ancestral mammalian $14 / 15$ form to $14 / 15 \mathrm{a}$ and $15 \mathrm{~b}$ in the ancestral Platyrrhini karyotype.

Despite the occurrence of extensive inter- and intrachromosomal rearrangements within the platyrrhines, the presence of a HSA 14/15 syntenic association in all species and subspecies studied so far confirms that this karyotypic feature is the ancestral condition of this group of primates. Furthermore, its presence in platyrrhines appears to be the retention of the pattern presumed to be the primitive mammalian pattern.

The HSA $14 / 15$ syntenic association is present in all primates except in the lineage leading to apes. Chromosome fission after the divergence of Old World monkeys has led to the separation of HSA 14 and 15 (Haig, 1999; Wienberg, 2005; Ferguson-Smith and Trifonov, 2007).

In humans, chromosome 15 shows a high degree of genomic instabilities, particularly in the proximal region, which has been found to be associated with various genomic diseases, as a consequence of deletions, duplications, translocations and inter- and intrachromosomal rearrangements in this region.

\section{Acknowledgments}

We would like to thank the Fundação Parque Zoológico de São Paulo (São Paulo, Brazil), Zoológico Municipal Quinzinho de Barros (Sorocaba, Brazil), Fundação Zôo-Botânica de Belo Horizonte (Belo Horizonte, Brazil), Departamento de Parques e Áreas Verdes do Estado de São Paulo, Divisão Técnica de Medicina Veterinária e Manejo da Fauna Silvestre (São Paulo, Brazil), Dr. Paulo Auricchio (Callicebus and Cacajao), Msc. Patrícia Beloto Bertola (Callithrix), Carlos Yamashita, Dr. Daniel Antônio Salati Marcondes and Dr. Sílvio Marsaru Ichihara (Alouatta caraya) for providing blood samples, and we truly appreciated the critical review of Dr. Eugene E. Harris. This research was supported by grants from FAPESP, CEPID-FAPESP and CNPq.

\section{References}

Barros RMS, Nagamachi CY, Pieczarka JC, Rodrigues LRR, Neusser M, Oliveira EH, Wienberg J, Muniz JAPC, Rissino JD and Müller S (2003) Chromosomal studies in Callicebus donacophilus pallescens, with classic and molecular cytogenetic approaches: Multicolor FISH using human and Saguinus oedipus painting probes. Chromosome Res 11:327-334.

Breen M, Thomas R, Binns MM, Carter NP and Langford CF (1999) Reciprocal chromosome painting reveals detailed regions of conserved synteny between the karyotypes of the domestic dog (Canis familiaris) and human. Genomics 61:145-155.

Chowdhary BP, Raudsepp T, Frönicke L and Scherthan H (1998) Emerging patterns of comparative genome organization in some mammalian species as revealed by Zoo-FISH. Genome Res 8:577-589.

Consigliere S, Stanyon R, Koehler U, Agoramoorthy G and Wienberg J (1996) Chromosome painting defines genomic rearrangements between red howler monkey subspecies. Chromosome Res 4:264-270.

Consigliere S, Stanyon R, Koehler U, Arnold N and Wienberg J (1998) In situ hybridization (FISH) maps chromosomal homologies between Alouatta belzebul (Platyrrhini, Cebidae) and other primates and reveals extensive interchromosomal 
rearrangements between howler monkey genomes. Am J Primatol 46:119-133.

De Oliveira EHC, Neusser M, Figueiredo WB, Nagamachi C, Pieczarka JC, Sbalqueiro IJ, Wienberg $\mathrm{J}$ and Müller S (2002) The phylogeny of howler monkeys (Alouatta, Platyrrhini): Reconstruction by multicolor cross-species chromosome painting. Chromosome Res 10:669-683.

De Oliveira EHC, Neusser M, Pieczarka JC, Nagamachi C, Sbalqueiro IJ and Müller S (2005) Phylogenetic inferences of Atelinae (Platyrrhini) based on multi-directional chromosome painting in Brachyteles arachnoides, Ateles paniscus paniscus and Ateles b. marginatus. Cytogenet Genome Res 108:183-190.

Dumas F, Bigoni F, Stone G, Sineo L and Stanyon R (2005) Mapping genomic rearrangements in titi monkeys by chromosome flow sorting and multidirectional in-situ hybridization. Chromosome Res 13:85-96.

Ferguson-Smith MA and Trifonov V (2007) Mammalian karyotype evolution. Nat Rev Genet 8:950-962.

Frönicke L (2005) Origins of primate chromosomes - As delineated by Zoo-FISH and alignments of human and mouse draft genome sequences. Cytogenet Genome Res 108:122138 .

García F, Nogués C, García M, Egozcue J and Ponsà M (1999) Characterization of constitutive heterochromatin in Cebus apella (Cebidae, Primates) and Pan troglodytes (Hominidae, Primates): Comparison to human chromosomes. Am J Primatol 49:205-221.

García F, Nogués C, Ponsà M, Ruiz-Herrera A, Egozcue J and Caldés MG (2000) Chromosomal homologies between humans and Cebus apella (Primates) revealed by Zoo-FISH. Mamm Genome 11:399-401.

García F, Ruiz-Herrera A, Egozcue J, Ponsà M and García M (2002) Chromosomal homologies between Cebus and Ateles (Primates) based on Zoo-FISH and G-banding comparisons. Am J Primatol 57:177-188.

Gerbault-Serreau M, Bonnet-Garnier A, Richard F and Dutrillaux B (2004) Chromosome painting comparison of Leontopithecus chrysomelas (Callitrichine, Platyrrhini) with man and its phylogenetic position. Chromosome Res 12:691-701.

Haig D (1999) A brief history of human autosomes. Phil Trans R Soc Lond B 354:1447-1470.

Jauch A, Wienberg J, Stanyon R, Arnold N, Tofanelli S, Ishida T and Cremer T (1992) Reconstruction of genomic rearrangements in great apes and gibbons by chromosome painting. Proc Natl Acad Sci USA 89:8611-8615.

Morescalchi MA, Schempp W, Consigliere S, Bigoni F, Wienberg J and Stanyon R (1997) Mapping chromosomal homology between humans and the black-handed spider monkey by fluorescence in situ hybridization. Chromosome Res 5:527-536.

Mudry MD, Rahn IM and Solari AJ (2001) Meiosis and chromosome painting of sex chromosome systems in Ceboidea. Am J Primatol 54:65-78.

Müller S, Neusser M, O'Brien PCM and Wienberg J (2001) Molecular cytogenetic characterization of the EBV - Producing cell line B95-8 (Saguinus oedipus, Platyrrhini) by chromosome sorting and painting. Chromosome Res 9:689-693.

Nash WG, Wienberg J, Ferguson-Smith MA, Menninger JC and O'Brien SJ (1998) Comparative genomics: Tracking chromosome evolution in the family Ursidae using reciprocal chromosome painting. Cytogenet Cell Genet 83:182-192.

Neusser M, Stanyon R, Bigoni F, Wienberg J and Müller S (2001) Molecular cytotaxonomy of the New World monkeys (Platyrrhini) - Comparative analysis of five species by multi-color chromosome painting gives evidence for a classification of Callimico goeldii within the family of Callitrichidae. Cytogenet Cell Genet 94:206-215.

Richard F, Lombard M and Dutrillaux B (1996) Zoo-FISH suggests a complete homology between human and capuchin monkey (Platyrrhini) euchromatin. Genomics 36:417-423.

Ruiz-Herrera A, García F, Mora L, Egozcue J, Ponsà M and García M (2005a) Evolutionary conserved chromosomal segments in the human karyotype are bounded by unstable chromosome bands. Cytogenet Genome Res 108:161-174.

Ruiz-Herrera A, García F, Aguilera M, García M and Fontanals MP (2005b) Comparative chromosome painting in Aotus reveals a highly derived evolution. Am J Primatol 65:73-85.

Seuánez HN, Alves G and O'Brien SJ (1994) Gene mapping in the spider monkey (Ateles paniscus chamek). J Hered 85:466473.

Sherlock JK, Griffin DK, Delhanty JDA and Parrington JM (1996) Homologies between human and marmoset (Callithrix jacchus) chromosomes revealed by comparative chromosome painting. Genomics 33:214-219.

Stanyon R, Tofanelli S, Morescalchi MA, Agoramoorthy G, Ryder OA and Wienberg J (1995) Cytogenetic analysis shows extensive genomic rearrangements between red howler (Alouatta seniculus, Linnaeus) subspecies. Am J Primatol 35:171-183.

Stanyon R, Consigliere S, Müller S, Morescalchi A, Neusser M and Wienberg J (2000) Fluorescence in situ hybridization (FISH) maps chromosomal homologies between the dusky titi and squirrel monkey. Am J Primatol 50:95-107.

Stanyon R, Consigliere S, Bigoni F, Ferguson-Smith M, O'Brien PCM and Wienberg J (2001) Reciprocal chromosome painting between a New World primate, the woolly monkey, and humans. Chromosome Res 9:97-106.

Stanyon R, Bonvicino CR, Svartman M and Seuánez HN (2003) Chromosome painting in Callicebus lugens, the species with the lowest diploid number $(2 \mathrm{n}=16)$ known in primates. Chromosoma 112:201-206.

Stanyon R, Bigoni F, Slaby T, Müller S, Stone G, Bonvicino CR, Neusser M and Seuanez HN (2004) Multi-directional chromosome painting maps homologies between species belonging to three genera of New World monkeys and humans. Chromosoma 113:305-315.

Wienberg J (2005) Fluorescence in situ hybridization to chromosomes as a tool to understand human and primate genome evolution. Cytogenet Genome Res 108:139-160.

\section{Associate Editor: Yatiyo Yonenaga-Yassuda}

License information: This is an open-access article distributed under the terms of the Creative Commons Attribution License, which permits unrestricted use, distribution, and reproduction in any medium, provided the original work is properly cited. 